\title{
Cervical Nerve Root Stimulation: DEMONSTRATION OF AN EXTRA-Foraminal TECHNIQUE
}

\author{
Frank J. E. Falco, MD, Daniel Kim, MD, and C. Obi Onyewu, MD
}

Since the description of electrical stimulation of nerve trunks as a means of providing pain relief, innovative peripheral nerve and nerve root stimulations have been developed. An extra-foraminal technique of electrical stimulation of cervical nerve roots resulting in reduction of up- per extremity radicular pain is demonstrated here. Electrical stimulation of the $\mathrm{C} 6$ and $\mathrm{C}_{7}$ nerve roots was accomplished using a four-electrode spinal cord stimulator lead that was placed percutaneously within the exit zone of the neuroforamina using an extra-foraminal approach. Subsequent
C6 and $\mathrm{C}_{7}$ nerve root stimulation provided a reduction in arm pain within the distribution of the stimulation.

Keywords: spinal cord stimulation, cervical radiculopathy, nerve root stimulation, pain, dorsal column stimulation, peripheral nerve stimulation.
In 1967, Shealy successfully implanted the first spinal cord stimulator for the treatment of chronic pain (1). The mechanism of pain relief through electrical stimulation is not clearly understood, but is believed to result from inhibition of pain transmission. There are several proposed theories for pain inhibition including antidromic activation of A-beta efferent nerve fibers; supraspinal inhibition; interruption of transmission through the spinothalamic tracts; central inhibition of the sympathetic efferents; and inhibition via the activation of neurotransmitters or neuromodulators (1-6).

Spinal cord (dorsal column) stimulation (SCS) is commonly used for the treatment of a variety of painful disorders such as peripheral vascular disease, radiculopathy, phantom limb pain, peripheral neuropathy, and complex regional pain syndromes (7-10). The most common indication in the United States for SCS is the treatment of radiculopathy secondary to failed back or neck syndrome and complex regional pain syndromes (11). Spinal cord stimulation is more effective in treating neuropahtic pain as opposed to primarily nociceptive pain disorders (12). Patients with distal extrem-

From Mid-Atlantic Spine, Newark, DE. Address Correspondence: Frank Falco, MD, Mid-Atlantic Spine, 139 East Chestnut Hill Road, Newark, DE-19713 Email: cssmo1@aol.com

Funding: There was no external funding in preparation of this manuscript.

Conflict of Interest: None ity pain respond better to SCS than those with predominately axial pain $(13,14)$. Spinal cord stimulation is not indicated for the treatment of individuals who suffer only from neck or back pain.

Spinal cord stimulation provides electrical current to the dorsal columns, which leads to paresthesias of the effected limb and subsequent pain reduction. Unfortunately, SCS is not always successful in treating the painful disorder due to an inability to capture the area of pain. In addition, SCS has been ineffective in treating axial related pain. The recent development of peripheral nerve and nerve root stimulation techniques using SCS technology have been successful in treating some painful axial disorders and radicular pain that has failed to respond to SCS $(10,15,16)$.

In 1959, Althaus described electrical stimulation of nerve trunks as a means of providing pain relief (17). Innovative peripheral nerve and nerve root stimulation techniques have been developed since that time for the treatment of painful disorders and other conditions not amenable or responsive to SCS. These techniques have provided relief for occipital headaches, pelvic pain, radicular pain, interstitial cystitis, trigeminal and postherpetic neuralgias, urinary urge incontinence, urinary retention and bladder detrusor dysfunction (7-10, 15, 16,18-23).

Peripheral nerve root stimulation has been used clinically for the treatment of pelvic pain, lumbar radiculopathy and several bladder disorders. Retrograde (craniocaudad) SCS electrode placement along lumbar and sacral nerve roots has been effective in the treatment of lumbar radiculopathy, pelvic pain, urge incontinence and bladder detrusor dysfunction $(15,18-20)$. Anterograde placement of SCS electrodes through the S2 foramen has been the primary stimulation method used for the treatment of non-obstructive urinary retention, urge incontinence and bladder detrusor dysfunction (21-23).

A trans-spinal approach in cadavers has been described for stimulation of cervical nerve roots (24). Electrodes were placed along the C5 through T2 nerve roots by advancing SCS leads laterally from the contralateral side of the cervical spine across the posterior epidural space into the cervical foramen. For C3 and C4 nerve roots a similar approach was used with a cephalocaudad approach to the foramen as opposed to the lateral method used for the lower cervical nerve roots in order to avoid the occiput, vertebral artery and cervical pelxus.

This report describes an extra-spinal, extra-foraminal nerve root stimulation (EFNRS) technique for the treatment of chronic cervical radicular pain. The stimulation of the cervical nerve roots provided paresthesias in the area of pain with a reduction in pain intensity. The patient tolerated the stimulation without complications or side effects. 


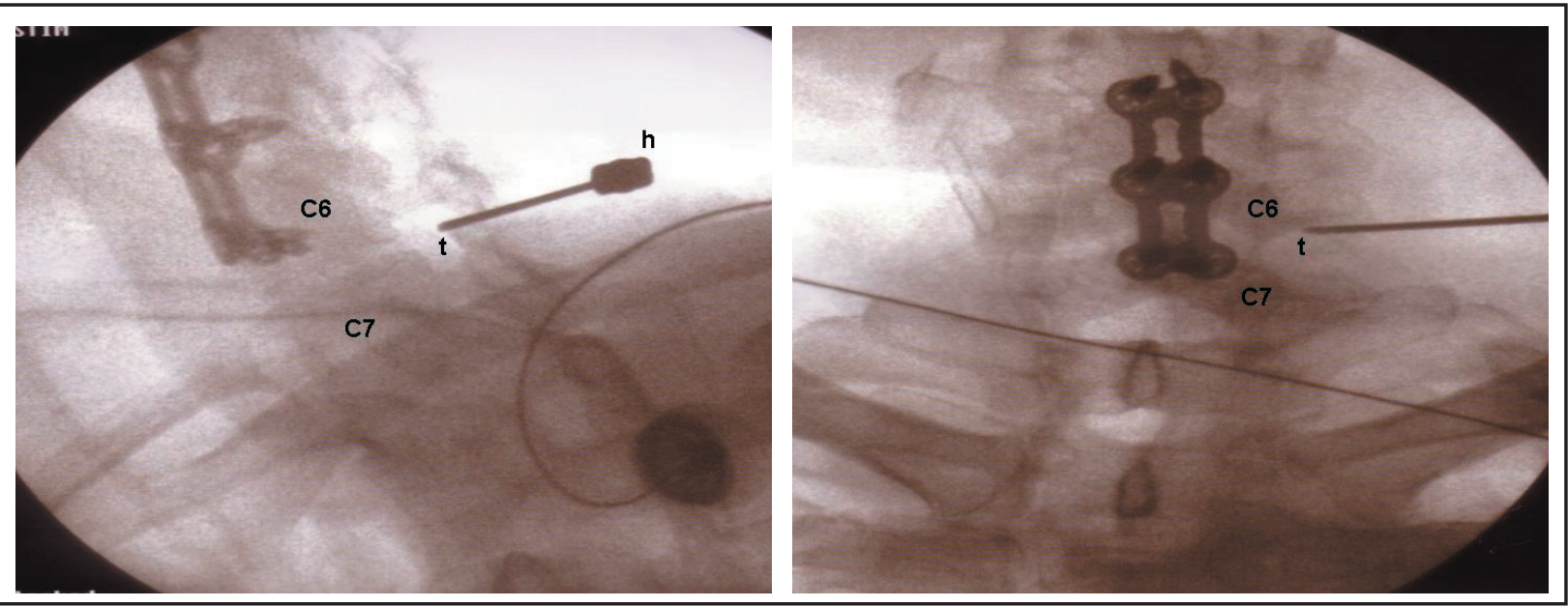

Fig 1. Needle placement into the eixt zone of the C6/7 cervical foramen. A. Oblique view. B. Posteroanterior (PA) view.

\section{DESCRIPTION OF TECHNIQUE}

\section{Background Information}

A 59-year old man with a chief complaint of numbness and burning in the left upper extremity was seen in consultation for pain management. The patient was involved in a motor vehicle accident four years ago sustaining a cervical spine hyperextension. He failed conservative treatment including medications and physical therapy. A cervical spine MRI revealed intervertebral disc herniations at the $\mathrm{C} 4 / 5$ and $\mathrm{C} 5 / 6$ levels with underlying central spinal stenosis. He subse-

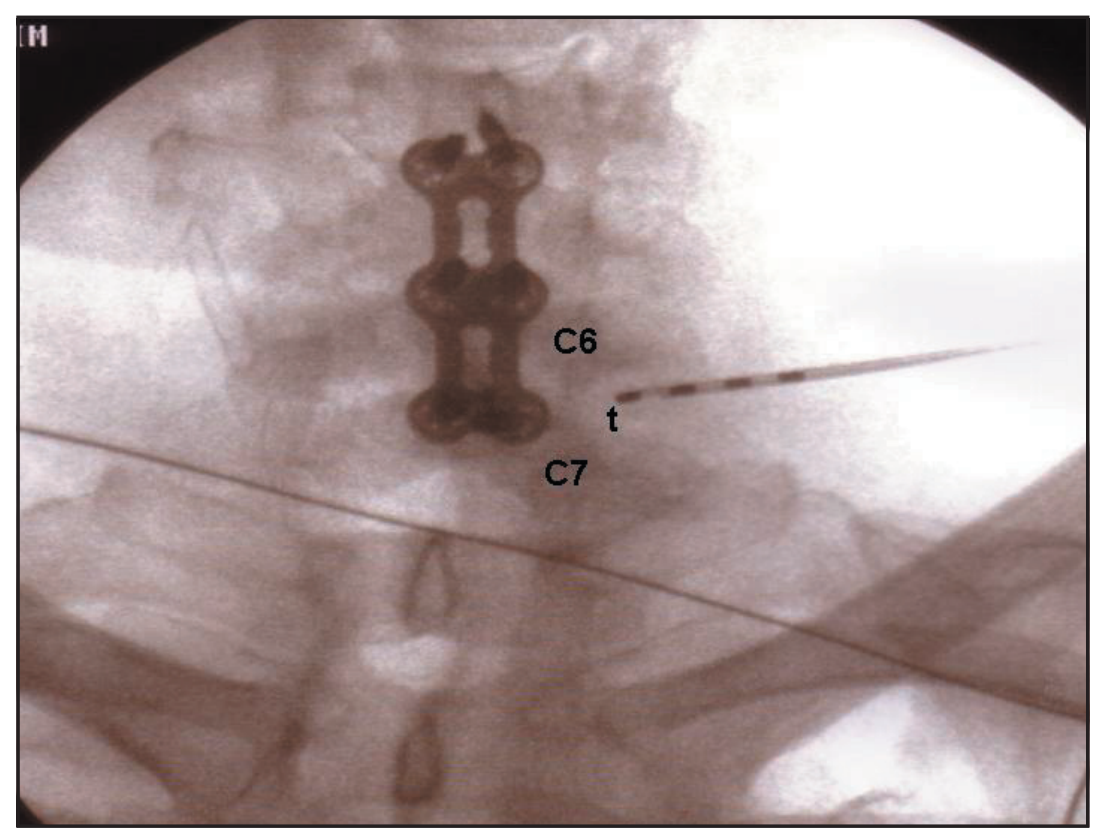

Fig 2. SCS lead within the needle after advancement to the needle tip. quently underwent a cervical fusion at the C4/5 and C5/6 levels without relief of his extremity symptoms. Post surgery he received a cervical epidural and a left C7 selective nerve root injection with temporary relief.

At the time of the consultation he complained of burning pain present in the left forearm extending into the $2^{\text {nd }}$ and $3^{\text {rd }}$ digits of his left hand. The pain was described as constant with an intensity of 67 on a numerical scale of 10 . There was also associated numbness in the same distribution of his pain. He was taking narcotic analgesics along with adjuvant neu-

ropathic agents for pain control with little relief of his symptoms. The patient underwent a cervical sympathetic block after the initial consultation with temporary relief of his symptoms lasting several days. Spinal cord stimulation was discussed with the patient at the follow up visit and he consented to an SCS trial.

An attempt was made to place a SCS lead into the posterior epidural space, but the trial was unsuccessful. The lead could not be advanced cephalad beyond the C6 vertebral level due to the spinal stenosis. An extra-foraminal technique was employed with electrical stimulation at the left C6 and subsequently the left C7 nerve roots providing paresthesias in their respective areas of innervation. Stimulation of the left $\mathrm{C} 7$ nerve root provided the patient with coverage and relief of his symptoms and the lead remained within the $\mathrm{C6/7}$ foramen for the trial. The patient had 50\% relief of his symptoms during the trial period but elected not to undergo percutaneous implantation. He was not interested in considering lead implantation using an open surgical technique.

\section{Technique for EFNRS}

The patient was placed in a supine position on the procedure table after informed and signed consent. The cervical, supraclavicular and chest areas were prepped and draped in the typical sterile fashion. Intravenous conscious sedation was induced prior to starting the procedure.

The cervical foramina were identified using a left anterolateral approach under fluoroscopic visualization. A modified 14 gauge epidural (Touhy ${ }^{\circledR}$ ) 


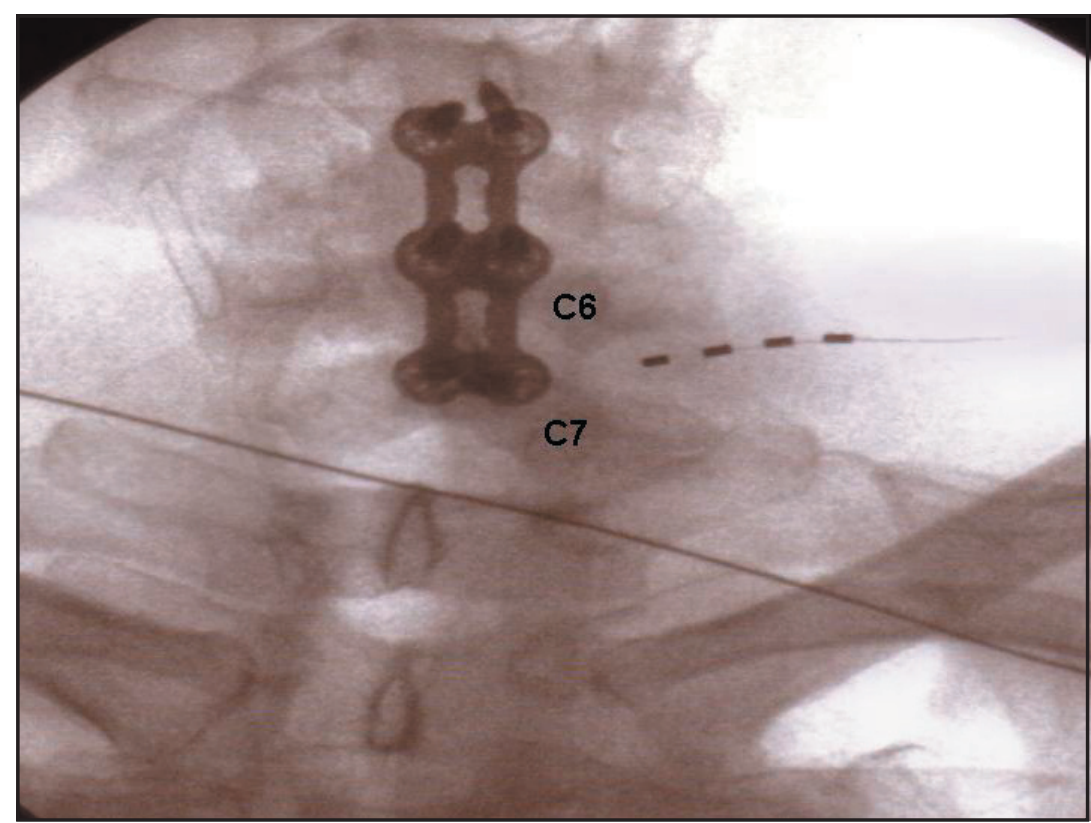

Fig 3. SCS lead within the C6/7 foramen after removal of the needle.

needle was advanced into the posterolateral aspect of the exit zone within the foramen, parallel to the nerve roots (Fig 1). Care was taken to avoid contact with the exiting nerve root and vertebral artery. A four electrode SCS lead (Quadtrode ${ }^{\circledR}$, Advanced Neuromodulation Systems, Inc, Plano, TX) was then passed through the needle and advanced to the needle tip (Fig 2). The needle was then removed leaving the electrode in place within the foramen, posterior and parallel to the nerve root (Fig 3). This method was used in sequence for placing the SCS lead within the left C5/6 and $\mathrm{C} 6 / 7$ foramina to stimulate the left $\mathrm{C} 6$ and $\mathrm{C} 7$ nerve roots respectively. Electrical stimulation of the $\mathrm{C} 7$ nerve root provided the best paresthesias and most relief of his symptoms in the distal left upper extremity. The lead was then secured to the skin for the trial using steri-strips and sterile clear bioadhesive.

\section{DisCussion}

Nerve root stimulation techniques have been described for lumbar and sacral nerve root stimulation using retrograde and anterograde techniques for the treatment of lumbosacral radiculopathy and pelvic pain $(15,16)$. An S2 transforaminal technique has been developed for the treatment of bladder disorders (21-23). Trans-spinal techniques for electrode lead placement along cervical nerve roots have been described in cadavers (24). The extra-foraminal technique described herein represents an alternative approach for nerve root stimulation in the treatment of cervical radiculopathy.

Conditions that may preclude a cervical epidural trial include previous spinal surgery, central canal stenosis or other spinal anomalies. The EFNRS technique may be an option, when an epidural approach is not possible, to trial an individual with upper extremity radicular pain prior to making a clinical decision regarding an open surgical SCS implantation. The EFNRS technique may even provide an alternative stimulation technique for treating cervical radiculopathy that does not respond to spinal cord stimulation as has been previously demonstrated with retrograde and anterograde nerve root stimulation methods in the treatment of lumbosacral pain. procedure include injury to the spinal cord, cervical nerve root, vertebral artery, spinal accessory nerve or carotid artery during needle placement. Pneumothorax is another potential complication when attempting EFNRS for C8 or T1 nerve root stimulation. A relative contraindication for this procedure may be foraminal stenosis, which might increase the risk for nerve root or vertebral artery injury. Obese patients or those with relatively "short" necks may also pose a relative contraindication, with a potential increased risk for the above complications.
These potential risks and relative contraindications described herein may be reduced with the use of introducer needles and electrodes that are smaller in diameter.

\section{Conclusions}

Extra-foraminal nerve root stimulation (EFNRS) is a percutaneous technique for electrode lead placement to stimulate cervical nerve roots for the treatment of upper extremity radicular pain. This technique may be an alternative electrical stimulation trial method for chronic cervical radiculopathy and other upper extremity pain syndromes, when a percutaneous epidural approach is not possible. Further investigation of this technique is warranted regarding the efficacy of this procedure and its possible use as an alternative implantation technique.

\section{ACKNOWLEDGEMENTS}

The authors wish to thank Bryan

Potential risks associated with this
Williams from Advanced Neuromodulation Systems, Inc.

\section{Author Affiliation: \\ Frank J. E. Falco, MD \\ Mid-Atlantic Spine \\ 139 East Chestnut Hill Road \\ Newark, DE-19713 \\ Email: cssm01@aol.com \\ Daniel Kim, MD \\ Mid-Atlantic Spine \\ 139 East Chestnut Hill Road \\ Newark, DE-19713 \\ C. Obi Onyewu, MD \\ Mid-Atlantic Spine \\ 139 East Chestnut Hill Road \\ Newark, DE-19713}

\section{REFERENCES}

1. Shealy CN, Mortimer JT, Resnick JB. Electrical inhibition of pain by stimulation of the dorsal columns: Preliminary reports. Anesth Analg 1967;46:489-491.

2. Feldman RA. Patterned response of lamina V cells: Cutaneous and dorsal funicular stimulation. Physiol Behav 1975;15:79-84.

3. Foreman RD, Beall JE, Coulter JD et al. Effects of dorsal column stimulation on primate spinothalamic tract neurons. I Neurophysiol 1976;39:534-546.

4. Handwerker HO, Iggo A, Zimmerman M. Segmental and supraspinal actions on ious and non-noxious skin stimuli. Pain 1975;1:147-165. dorsal horn neurons responding to nox- 
5. Krames ES: Mechanissms of action of spinal cord stimulation. In Waldman SD, Winnie AP (eds): Interventional Pain Management. WB Saunders, Philadelphia, 1996 , pp. 407-411.

6. Melzack R, Wall PD. Pain mechanism: A new theory. Science 1965;150:971-979.

7. North RB, Kidd DH, James C, Long DM. Spinal cord stimulation for chronic intractable pain: Experience over two decades. Neurosurgery 1993;32:384-395.

8. Burchiel KJ, Anderson VC, Brown FD et al. Prospective multicenter study of spinal cord stimulation for relief of chronic back and extremity pain. Spine 1996;21:27862794 .

9. Al_KM and Holsheimer J. New trends in neuromodulation for the management of neuropathic pain. Neurosurgery 2002;50: 690-703.

10. Deer TR. Current and future trends in spinal cord stimulation for chronic pain. Curr Pain Headache Rep 2001;5:503-509.

11. North RB, Wetzel FT. Spinal cord stimulation for chronic pain of spinal origin. Spine 2002;27:2584-2591.

12. Oakley J, Prager J. Spinal cord stimulation: Mechanism of action. Spine 2002; 27: 2574-2583.
13. North RB, Kidd DH, Lee MS et al. A prospective, randomized study of spinal cord stimulation vs. reoperation for failed back surgery syndrome: Initial results. Sterotact Funct Neurosurg 1994;62:267272.

14. Turner J, Loeser JD, Bell KG. Spinal cord stimulation for chronic low back pain: $A$ systematic literature synthesis. Neurosurgery 1995;37:1088-1096.

15. AlóKM, Yland MJ, Redko V, Feler CA, Naumann C. Lumbar and sacral nerve root stimulation (NRS) in the treatment of chronic pain: a novel anatomic approach and neuro stimulation technique. Neuromodulation 1999;2:23-31.

16. Falco FJE, Rubbani M, Heinbaugh J. Anterograde sacral nerve root stimulation (ASNRS) via the sacral hiatus: Benefits, limitations, and percutaneous implantation technique. Neuromodulation In Press.

17. Althaus J. A treatise of medical electricity, theoretical and practical and its use in the treatment of paralysis. In: Neuralgia and other disease. Trubner, London, 1959.

18. Feler CA, Whitworth LA, Brookoff $D$ et al. Recent advances: sacral nerve root stimulation using a retrograde method of lead insertion for the treatment of pelvic pain due to interstitial cystitis. Neurmodulation 1999;2:211-216.

19. Al_KM, Mckay E. Selective nerve root stimulation (SNRS) for the treatment of intractable pelvic pain and motor dysfunction. A case report. Neuromodulation 2001;4:19-23.

20. Al_KM, Gohel R, Corey C. Sacral nerve root stimulation for the treatment of urge incontinence and detrusor dysfunction utilizing a cephalocaudal intraspinal method of lead insertion: A case report. Neuromodulation 2001;4:53-58.

21. Shaker HS, Hassouna M. Sacral root neuromodulation in idiopathic non-obstructive chronic urinary retention. J Urol 1998;159:1476-1478.

22. Shaker HS, Hassouna M. Sacral nerve root neuromodulation: an effective treatment of refractory urge incontinence. J Uro 1998;159:1516-1519.

23. Robin S, Sawan M, Abdel-Gawad M et al. Implantable stimulation system dedicated for neural selective stimulation. Med Biol Eng Comput 1998;36:490-492.

24. AlóKM, Yland MJ, Feler C et al. A study of electrode placement at the cervical and upper thoracic nerve roots using an anatomic trans-spinal approach. Neuromod ulation 1999;2:222-227. 